\title{
On Stress Analysis for Cracks in Elastic Materials with Voids
}

\author{
M. Ciarletta, G. Iovane \\ D.I.I.M.A., University of Salerno, \\ Via Ponte don Melillo, 84084 Fisciano (SA), Italy \\ e-mail: ciarlett@diima.unisa.it \\ e-mail: iovane@diima.unisa.it \\ and \\ M. A. Sumbatyan \\ Rostov State University, Faculty of Mechanics and Mathematics, \\ Zorge Street 5, Rostov-on-Don 344090, Russia \\ e-mail: sumbat@math.rsu.ru
}

\begin{abstract}
The paper deals with classical problem for cracks dislocated in a certain very specific porous elastic material, described by a Cowin-Nunziato model. We propose a method based upon a reducing of stress concentration problem for cracks to some integral equations. By applying Fourier integral transforms the problem is reduced to some integral equations. For the plane-strain problem we operate with a direct numerical treatment of a hypersingular integral equation. In the axially symmetric case, for the penny-shaped crack, the problem is reduced to a regular Fredholm integral equation of the second kind. In the both cases we study stress-concentration factor, and investigate its behavior versus porosity of the material. More in particular the stress concentration factor in the medium with voids is always higher, under the same conditions, than in the classical elastic medium made of material of the skeleton. Further,
\end{abstract}


as can be seen, the influence of the porosity becomes more significant for larger cracks; that is also quite natural from a physical point of view.

\section{Introduction}

There are a number of theories about mechanical properties of porous materials. One of them is a Biot consolidation theory of fluid-saturated porous solids [1]. Typically, these theories reduce to classical elasticity when the pore fluid is absent. This is why Cowin and Nunziato proposed a new theory to describe properties of homogeneous elastic materials with voids free of fluid [2]. This theory is a special case of microstretch elasticity of Eringen, when micropolar effects are discarded. Eringen's microstretch theory is more appropriate for geological materials like rocks, soils since, this theory takes into account the intrinsic rotations and stretch of materials [5], [6]. These effects are not considered in Cowin-Nunziato theory. However, the last theory is mathematically simpler to deal with, and it takes into account voids in porous materials without any other phase, like liquid and gas.

A general theory of such materials is currently well-developed by many authors (see, for example [3],[4]), but too few concrete problems are solved, to allow one to estimate the practical merits of this model.

Generally, this theory is founded on the balance of energy, where presence of the pores involves additional degree of freedom, namely, the fraction of elementary volume. As a consequence, the bulk mass density is given by the product of two fields, the void volume fraction and the mass density of the matrix (elastic) material.

An exact explicit solution is well known in the problem for a crack (both linear and penny-shaped) dislocated in the classical linear elastic space [9]. If a normal load is applied to the faces of the crack, then the shape of the faces near the edge of the crack under this stress can be represented explicitly as a rootsquare function. This permits analytical calculation of the stress concentration coefficient in the classical case. Obviously, stress concentration analysis is also very important in engineering practice for porous materials. Therefore, the main goal of the present work is to construct a strict solution of the static crack problem for the line (plane-strain problem) and penny shaped (axially symmetric problem) cracks dislocated in the linear elastic granular (porous) space. 
We first give a short summary of basic equations, then demonstrate application of the Fourier transform that allows us to reduce the problem to some integral equations and to construct a direct numerical collocation technique to solve this equation. In the plane-strain problem we treat numerically a certain hypersingular integral equation, and a special kind of the collocation technique can be also applied to such equations. Finally, we demonstrate in figures how the calculated stress concentration coefficient at the crack edge depends upon variation of some physical and geometric parameters.

\section{Governing Equations for Elastic Media with Voids: Plane-Strain Problem}

We refer the deformation of the continuum to a fixed system of rectangular Cartesian coordinates $O x y z$. Let us consider elastic material with voids which possesses a reference configuration with a constant volume fraction $\nu_{0}$. The considered theory asserts that the constant mass density $\varrho$ has the decomposition $[2] \varrho=\gamma \nu$, where $\gamma$ is the density of the matrix material, and $\nu \quad(0<\nu \leq 1)$ is the volume fraction field.

Let $\phi\left(\phi=\nu-\nu_{0}\right)$ be the change in volume fraction from the reference one. Then the linear theory of homogeneous and isotropic elastic material with voids is described by the following system of partial differential equations $[2,8]$

$$
\left\{\begin{array}{c}
\mu \Delta \bar{u}+(\lambda+\mu) \operatorname{grad} \operatorname{div} \bar{u}+\beta \operatorname{grad} \phi=0 \\
\alpha \Delta \phi-\xi \phi-\beta \operatorname{div} \bar{u}=0
\end{array}\right.
$$

where $\mu$ and $\lambda$ are classical elastic constants; $\alpha, \beta$ and $\xi$ - some constants related to porosity of the medium. Besides, $\bar{u}$ denotes the displacement vector. Obviously, if $\beta=0$, then the elastic and the "porosity" fields are independent. Thus, in the case $\beta=0$ the stress-strain state is insensitive to the function $\phi$. The components of the stress tensor are defined, in terms of the functions $\bar{u}$ and $\phi$, by the following relations ( $\delta_{i j}$ is the Kronecker's delta)

$$
\left\{\begin{aligned}
\sigma_{i j} & =\lambda \delta_{i j} \varepsilon_{k k}+2 \mu \varepsilon_{i j}+\beta \phi \delta_{i j} \\
\varepsilon_{i j} & =\frac{1}{2}\left(u_{i, j}+u_{j, i}\right)
\end{aligned}\right.
$$


Let us formulate the plane-strain boundary value problem for the porous (granular) medium. In this case $\bar{u}=\left\{u_{x}(x, y), u_{y}(x, y), 0\right\}$, and the basic system (2.1) can be rewritten as follows

$$
\left\{\begin{array}{l}
\frac{\partial^{2} u_{x}}{\partial x^{2}}+c^{2} \frac{\partial^{2} u_{x}}{\partial y^{2}}+\left(1-c^{2}\right) \frac{\partial^{2} u_{y}}{\partial x \partial y}+H \frac{\partial \phi}{\partial x}=0 \\
\frac{\partial^{2} u_{y}}{\partial y^{2}}+c^{2} \frac{\partial^{2} u_{y}}{\partial x^{2}}+\left(1-c^{2}\right) \frac{\partial^{2} u_{x}}{\partial x \partial y}+H \frac{\partial \phi}{\partial y}=0 \\
l_{1}^{2}\left(\frac{\partial^{2} \phi}{\partial x^{2}}+\frac{\partial^{2} \phi}{\partial y^{2}}\right)-\frac{l_{1}^{2}}{l_{2}^{2}} \phi-\left(\frac{\partial u_{x}}{\partial x}+\frac{\partial u_{y}}{\partial y}\right)=0
\end{array},\right.
$$

with

$$
\begin{gathered}
\frac{\sigma_{x x}}{\lambda+2 \mu}=\frac{\partial u_{x}}{\partial x}+\left(1-2 c^{2}\right) \frac{\partial u_{y}}{\partial y}+H \phi, \\
\frac{\sigma_{y y}}{\lambda+2 \mu}=\left(1-2 c^{2}\right) \frac{\partial u_{x}}{\partial x}+\frac{\partial u_{y}}{\partial y}+H \phi, \\
\frac{\sigma_{x y}}{\mu}=\frac{\partial u_{x}}{\partial y}+\frac{\partial u_{y}}{\partial x} .
\end{gathered}
$$

The positive physical parameters (2.3)-(2.4) are introduced as follows

$$
c^{2}=\frac{\mu}{\lambda+2 \mu}, \quad H=\frac{\beta}{\lambda+2 \mu}, \quad l_{1}^{2}=\frac{\alpha}{\beta}, \quad l_{2}^{2}=\frac{\alpha}{\xi},
$$

where the first two numbers $c, H$ are dimensionless and the quantities $l_{1}, l_{2}$ have the dimension of length.

Let us consider a thin crack of the length $2 a$ with plane faces, dislocated over the segment $-a<x<a$ along the $x$-axis. Let the plane-strain deformation of this crack be caused by a constant stress $\sigma_{y y}=\sigma_{0}$ applied at infinity (i.e. at $y= \pm \infty)$. Then, due to linearity of the problem, it is easily seen that both the shape of the crack's faces and the stress concentration at the crack's edges are the same as in the problem with a solution decaying at infinity and the following boundary conditions corresponding to the case when the normal load $-\sigma_{0}$ is symmetrically applied to the faces of the crack and there is no load at infinity.

For the last problem the boundary conditions over the line $y=0$ are

$$
\sigma_{x y}=0, \frac{\partial \phi}{\partial y}=0(|x|<\infty), \quad \sigma_{y y}=-\sigma_{0}(|x|<a), \quad u_{y}=0(|x|>a) .
$$


It is proved $(\mathrm{cf} .[7,10])$ that the boundary condition for the function $\phi(2.6)$ directly follows from a balance principle.

Let us apply the Fourier transform along the x-axis to relations $(2.3),(2.4)$ and the boundary conditions (2.6). Then Eqs.(2.3) reduce to the following system of ordinary differential equations, with respect to images of the functions $u_{x}, u_{y}, \phi$. Therefore, these become functions of the variable $y$ only, with the Fourier parameter $s$ instead of the first variable $x$ (all Fourier images are denoted by respective capital letters, for all physical quantities):

$$
\left\{\begin{array}{l}
c^{2} U_{x}^{\prime \prime}-s^{2} U_{x}+\left(1-c^{2}\right)(-i s) U_{y}^{\prime}-i s H \Phi=0 \\
\left(1-c^{2}\right)(-i s) U_{x}^{\prime}+U_{y}^{\prime \prime}-c^{2} s^{2} U_{y}+H \Phi^{\prime}=0 \\
i s U_{x}-U_{y}^{\prime}+l_{1}^{2} \Phi^{\prime \prime}-\left(\frac{l_{1}^{2}}{l_{2}^{2}}+l_{1}^{2} s^{2}\right) \Phi=0 .
\end{array}\right.
$$

Let us introduce the new unknown function $g(x)$ as

$$
u_{y}(x, 0)=\left\{\begin{array}{cc}
g(x), & |x|<a \\
0, & |x|>a
\end{array},\right.
$$

then the boundary conditions (2.6) in Fourier images become

$$
U_{x}^{\prime}-i s U_{y}=0, \quad \Phi^{\prime}=0, \quad U_{y}=G(s), \quad y=0
$$

where

$$
G(s)=\int_{-a}^{a} g(\xi) \exp (i s \xi) d \xi .
$$

General solution of the system (2.7) is constructed in accordance with a classical theory of ordinary differential equations (cf.[10]). Due to natural symmetry of the problem with respect to $x$-axis, we give here only solution for $y \geq 0$, which has the following form (here all physical quantities of dimension of length, including components of the displacement vector, are written in a 
dimensionless form being related to $l_{2}$ )

$$
\begin{gathered}
\left(\begin{array}{c}
U_{x} \\
U_{y} \\
\Phi
\end{array}\right)=D_{1}\left(\begin{array}{c}
\frac{i s H}{1-N} \\
\frac{H q(s)}{1-N} \\
1
\end{array}\right) e^{-q(s) y}+D_{2}\left(\begin{array}{c}
i \operatorname{sign}(s) \\
1 \\
0
\end{array}\right) e^{-|s| y}+ \\
D_{3}\left(\begin{array}{c}
\frac{1-N+c^{2}}{(-i s)\left(N-1+c^{2}\right)}+i y \operatorname{sign}(s) \\
y \\
\frac{2 N c^{2}}{H\left(1-N-c^{2}\right)}
\end{array}\right) e^{-|s| y}, q(s)=\sqrt{s^{2}+1-N},
\end{gathered}
$$

where $N=\left(l_{2}^{2} / l_{1}^{2}\right) H, \quad 0 \leq N<1$ is the so-called "coupling number". Obviously, the unknown constants $D_{1}, D_{2}, D_{3}$ should be defined from boundary conditions (2.9a). These yield the $3 \times 3$ algebraic system

$$
\left\{\begin{array}{l}
\frac{s H q(s)}{1-N} D_{1}+s D_{2}+\frac{(1-N) \operatorname{sign}(s)}{N-1+c^{2}} D_{3}=0 \\
q(s) D_{1}+\frac{2 N c^{2}|s|}{H\left(1-N-c^{2}\right)} D_{3}=0 \\
\frac{H q(s)}{1-N} D_{1}+D_{2}=G(s)
\end{array}\right.
$$

whose principal determinant is

$$
\Delta=\frac{(1-N) q(s) \operatorname{sign}(s)}{N-1+c^{2}} .
$$

The particular ones (related to the three unknowns when one applies the Cramer's rule) are:

$$
\begin{gathered}
\Delta_{1}=-G(s) \frac{2 N c^{2} s|s|}{H\left(N-1+c^{2}\right)} \\
\Delta_{2}=G(s) \frac{q(s) \operatorname{sign}(s)\left[(1-N)^{2}+2 N c^{2} s^{2}\right]}{(1-N)\left(N-1+c^{2}\right)}
\end{gathered}
$$




$$
\Delta_{3}=-G(s) q(s) s .
$$

Thus, the Fourier image $P(s)$ of the normal stress $\sigma_{y y}$ over the line $y=0$ as follows

$$
\begin{gathered}
\frac{P(s)}{2 \mu}=\frac{H s^{2}}{1-N} D_{1}+|s| D_{2}+\frac{c^{2}}{N-1+c^{2}} D_{3}= \\
=\frac{G(s)|s|}{(1-N)^{2} q(s)}\left[2 N c^{2} s^{2}(q-|s|)+(1-N)\left(1-N-c^{2}\right) q\right] .
\end{gathered}
$$

By applying Fourier inversion to relation (2.13), with the use of a convolution theorem, one can express the normal stress on the line of symmetry $y=0$ in terms of its opening:

$$
\frac{(1-N)^{2}}{2 \mu} \sigma_{y y}(x, 0)=\int_{-b}^{b} g(\xi) K(x-\xi) d \xi, \quad\left(b=\frac{a}{l_{2}}\right)
$$

where

$$
\begin{gathered}
K(x)=\frac{1}{2 \pi} \int_{-\infty}^{\infty} \frac{|s|}{q(s)}\left[2 N c^{2} s^{2}(q-|s|)+(1-N)\left(1-N-c^{2}\right) q\right] e^{-i s x} d s \\
=\frac{1}{\pi} \int_{0}^{\infty} L(s) \cos (s x) d s, \quad q=q(s)=\sqrt{s^{2}+1-N} \\
L(s)=\frac{s}{q(s)}\left[2 N c^{2} s^{2}(q-s)+(1-N)\left(1-N-c^{2}\right) q\right] .
\end{gathered}
$$

Finally, the boundary condition $\sigma_{y y}(x, 0)=-\sigma_{0}, \quad(|x|<a)$ from $(2.6)$ yields the main integral equation for the considered plane-strain problem with the convolution kernel

$$
\int_{-b}^{b} g(\xi) K(x-\xi) d \xi=-(1-N)^{2} \frac{\sigma_{0}}{2 \mu}, \quad|x|<b .
$$




\section{Reduction to Integral Equation in the Axi- ally Symmetric Case}

In this case the general equations (2.1) reduce, instead of Eqs.(2.3), to the following system of partial differential equations for the components of the displacement vector in cylindrical coordinates $\bar{u}=\left\{u_{r}(x, y), 0, u_{z}(x, y)\right\}$

$$
\left\{\begin{array}{l}
\left(\frac{\partial^{2} u_{r}}{\partial r^{2}}+\frac{1}{r} \frac{\partial u_{r}}{\partial r}-\frac{u_{r}}{r^{2}}\right)+c^{2} \frac{\partial^{2} u_{r}}{\partial z^{2}}+\left(1-c^{2}\right) \frac{\partial^{2} u_{z}}{\partial r \partial z}+H \frac{\partial \phi}{\partial r}=0 \\
\left(1-c^{2}\right) \frac{\partial}{\partial z}\left(\frac{\partial u_{r}}{\partial r}+\frac{u_{r}}{r}\right)+c^{2}\left(\frac{\partial^{2} u_{z}}{\partial r^{2}}+\frac{1}{r} \frac{\partial u_{z}}{\partial r}\right)+\frac{\partial^{2} u_{z}}{\partial z^{2}}+H \frac{\partial \phi}{\partial z}=0 \\
l_{1}^{2}\left(\frac{\partial^{2} \phi}{\partial r^{2}}+\frac{1}{r} \frac{\partial \phi}{\partial r}+\frac{\partial^{2} \phi}{\partial z^{2}}\right)-\frac{l_{1}^{2}}{l_{2}^{2}} \phi-\left(\frac{\partial u_{r}}{\partial r}+\frac{u_{r}}{r}+\frac{\partial u_{z}}{\partial z}\right)=0
\end{array}\right.
$$

with

$$
\begin{gathered}
\frac{\sigma_{r r}}{\lambda+2 \mu}=\frac{\partial u_{r}}{\partial r}+\left(1-2 c^{2}\right)\left(\frac{u_{r}}{r}+\frac{\partial u_{z}}{\partial z}\right)+H \phi \\
\frac{\sigma_{z z}}{\lambda+2 \mu}=\left(1-2 c^{2}\right)\left(\frac{\partial u_{r}}{\partial r}+\frac{u_{r}}{r}\right)+\frac{\partial u_{z}}{\partial z}+H \phi, \\
\frac{\sigma_{r z}}{\mu}=\frac{\partial u_{r}}{\partial z}+\frac{\partial u_{z}}{\partial r},
\end{gathered}
$$

If a round thin plane crack is placed at the center of the cylindrical coordinates: $z=0, r<a$ and its faces is under a normal constant load $-\sigma_{0}$, then the boundary conditions over the plane of symmetry $z=0$ are

$$
\sigma_{r z}=0, \frac{\partial \phi}{\partial z}=0(r<\infty), \quad \sigma_{z z}=-\sigma_{0}(r<a), \quad u_{z}=0(r>a) .
$$

Let us apply the Hankel transform along the $r$-variable to relations (3.1),(3.2):

$$
\begin{array}{ll}
U_{r}(s, z)=\int_{0}^{\infty} u_{r}(r, z) J_{1}(s r) r d r, \quad u_{r}(r, z)=\int_{0}^{\infty} U_{r}(s, z) J_{1}(s r) s d s, \\
U_{z}(s, z)=\int_{0}^{\infty} u_{z}(r, z) J_{0}(s r) r d r, \quad u_{z}(r, z)=\int_{0}^{\infty} U_{z}(s, z) J_{0}(s r) s d s
\end{array}
$$




$$
\phi(s, z)=\int_{0}^{\infty} \Phi(r, z) J_{0}(s r) r d r, \quad \Phi(r, z)=\int_{0}^{\infty} \phi(s, z) J_{0}(s r) s d s .
$$

If one applies the Hankel transform to equations (3.1), then one comes to the system of ordinary differential equations, with respect to images of the functions $u_{r}, u_{z}, \phi$. Therefore, these become functions of the variable $z$ only, with some parameter $s$ :

$$
\left\{\begin{array}{c}
c^{2} U_{r}^{\prime \prime}-s^{2} U_{r}-\left(1-c^{2}\right) s U_{z}^{\prime}-H s \Phi=0 \\
\left(1-c^{2}\right) s U_{r}^{\prime}+U_{z}^{\prime \prime}-c^{2} s^{2} U_{z}+H \Phi^{\prime}=0 \\
-s U_{r}-U_{z}^{\prime}+l_{1}^{2} \Phi^{\prime \prime}-\left(\frac{l_{1}^{2}}{l_{2}^{2}}+l_{1}^{2} s^{2}\right) \Phi=0
\end{array}\right.
$$

where all derivatives are applied with respect to the variable $z$.

Solution of Eqs.(3.5), together with Eqs.(3.4) and boundary conditions (3.3) gives for the Hankel image of the normal stress $\sigma_{z z}$ the following expression in terms of the image of crack's faces opening

$$
\frac{P(s)}{\mu}=\frac{G(s) s}{(1-N)^{2} q(s)}\left[2 N c^{2} s^{2}(q-s)+(1-N)\left(1-N-c^{2}\right) q\right],
$$

where

$$
u_{z}(r, 0)=\left\{\begin{array}{cc}
g(r), & r<b \\
0, & r>b
\end{array}\right.
$$

and

$$
G(s)=\int_{0}^{b} g(\rho) J_{0}(s \rho) \rho d \rho, \quad P(s)=\int_{0}^{\infty} \sigma_{z z}(\rho, 0) J_{0}(s \rho) \rho d \rho .
$$

If one applies inverse Hankel transform to relation (3.8) then one arrives at the main integral equation for the round crack problem:

$$
\int_{0}^{b} g(\rho) \rho K(r, \rho) d \rho=-(1-N)^{2} \frac{\sigma_{0}}{\mu}, \quad 0<r<b \quad\left(b=\frac{a}{l_{2}}\right)
$$

with the kernel

$$
K(r, \rho)=\int_{0}^{\infty} L(s) J_{0}(r s) J_{0}(\rho s) s^{2} d s
$$




\section{Properties of Integral Equations and Numer- ical Implementation for the Plane Problem}

Let us start from the evident estimate

$$
q(s) \sim s\left[1+\frac{1-N}{2 s^{2}}+O\left(\frac{1}{s^{4}}\right)\right] \text { at } s \rightarrow+\infty,
$$

so the asymptotic behavior of the symbolic function of the kernel at infinity is given as follows

$$
L(s) \sim(1-N)^{2}\left(1-c^{2}\right) s+O\left(\frac{1}{s}\right), \quad s \rightarrow \infty .
$$

It is obvious that the leading asymptotic term (4.2) leads to a principal degree of the kernel's (2.15) singularity, and one can see that the latter is defined by the integral

$$
\int_{0}^{\infty} s \cos (s x) d s=-\frac{1}{x^{2}},
$$

i.e. the kernel is hypersingular when $x \rightarrow 0$ [9]. It can be shown that the remaining term in asymptotic estimate (4.2) possesses a logarithmic (i.e. weak) singularity at $x \rightarrow 0$.

However, the kernel (2.15) admits explicit representation that permits direct estimate of its singular properties. To obtain such a representation, we calculate the following integrals

$$
\int_{0}^{\infty} s^{3} \cos (s x) d s=-\frac{d^{2}}{d x^{2}} \int_{0}^{\infty} s \cos (s x) d s=\frac{6}{x^{4}} .
$$

Then we consider the following table integral (here $K_{0}$ is a McDonald's function of the order 0 )

$$
\int_{0}^{\infty} \frac{\cos (s x)}{q(s)} d s=K_{0}(\sqrt{1-N}|x|),
$$

and apply, step by step, sequential derivatives to Eq.(4.5), as follows (assuming 
$x>0)$

$$
\begin{gathered}
\int_{0}^{\infty} \frac{s^{2} \cos (s x)}{q(s)} d s=-\frac{d^{2}}{d x^{2}}\left[K_{0}(\sqrt{1-N} x)\right]= \\
=\sqrt{1-N} \frac{d}{d x} K_{1}(\sqrt{1-N} x)=(1-N) \frac{d}{d(\sqrt{1-N} x)} K_{1}(\sqrt{1-N} x)= \\
=-\left[(1-N) K_{0}(\sqrt{1-N} x)+\frac{\sqrt{1-N}}{x} K_{1}(\sqrt{1-N} x)\right], \\
\int_{0}^{\infty} \frac{s^{4} \cos (s x) d s}{(1-N) q(s)}=\frac{d^{2}}{d x^{2}}\left[K_{0}(\sqrt{1-N} x)+\frac{K_{1}(\sqrt{1-N} x)}{\sqrt{1-N} x}\right]= \\
=2\left(\frac{3}{x^{3}}+\frac{1-N}{x}\right) \frac{K_{1}(\sqrt{1-N} x)}{\sqrt{1-N}}+\left(1-N+\frac{3}{x^{2}}\right) K_{0}(\sqrt{1-N} x),
\end{gathered}
$$

where we have used the derivatives of the McDonald's functions of the zero's and the first order:

$$
\frac{d K_{0}(z)}{d z}=-K_{1}(z), \quad \frac{d K_{1}(z)}{d z}=-K_{0}(z)-\frac{K_{1}(z)}{z} .
$$

Therefore, the full kernel for the plane-strain crack problem is explicitly represented as follows

$$
\begin{aligned}
& K(x)=\frac{1}{\pi}\left\{2 N c ^ { 2 } \left[\frac{6}{x^{4}}-2 \sqrt{1-N}\left(\frac{3}{|x|^{3}}+\frac{1-N}{|x|}\right) K_{1}(\sqrt{1-N}|x|)-\right.\right. \\
& \left.\left.-(1-N)\left(1-N+\frac{3}{x^{2}}\right) K_{0}(\sqrt{1-N}|x|)\right]-\frac{(1-N)\left(1-N-c^{2}\right)}{x^{2}}\right\} .
\end{aligned}
$$

¿From the last representation it is obvious that the function $K(x)(4.9)$ is differentiable as many times as wanted outside a small vicinity of the origin $x=0$. Let us estimate its behavior when $x \rightarrow 0$. For this aim one can use the following asymptotic formulas

$$
\begin{gathered}
K_{0}(z) \sim-\ln \left(\frac{z}{2}\right)-\gamma+O\left(z^{2} \ln z\right), \quad z \rightarrow+0, \\
K_{1}(z) \sim \frac{1}{z}+\frac{z}{2} \ln \left(\frac{z}{2}\right)+\left(\gamma-\frac{1}{2}\right) \frac{z}{2}+O\left(z^{3} \ln z\right), \quad z \rightarrow+0,
\end{gathered}
$$


where $\gamma=0.577216$ is the Euler's constant [10]. Thus behavior of function (4.9) for the small argument is

$$
K(x) \sim-\frac{(1-N)^{2}\left(1-c^{2}\right)}{\pi x^{2}}+O(\ln |x|), \quad x \rightarrow 0,
$$

so the kernel is hypersingular indeed.

The numerical method that we apply to solve the hypersingular integral equation has been proposed in our previous paper [9]. It is based on extraction of a characteristic hypersingular part of the kernel. Let us represent the full equation as follows

$$
\int_{-b}^{b}\left[\frac{1}{(x-t)^{2}}+K_{*}(x, t)\right] g(t) d t=f^{\prime}(x), \quad x \in(-b, b),
$$

where the regular part $K_{*}$ may admit a weak (i.e. integrable) singularity. If we represent it as

$$
K_{*}(x, t)=\frac{\partial K_{1}(x, t)}{\partial x},
$$

then a bounded solution can be constructed by applying inversion of the characteristic part, that reduces eq.(4.12) to a second-kind Fredholm integral equation

$$
g(x)+\int_{-b}^{b} N_{1}(x, t) g(t) d t=f_{1}(x), \quad x \in(-b, b),
$$

where

$$
\begin{gathered}
N_{1}(x, t)=\frac{\sqrt{b^{2}-x^{2}}}{\pi^{2}} \int_{-b}^{b} \frac{K_{1}(\tau, t) d \tau}{\sqrt{b^{2}-\tau^{2}}(x-\tau)}, \\
f_{1}(x)=\frac{\sqrt{b^{2}-x^{2}}}{\pi^{2}} \int_{-b}^{b} \frac{f(\tau) d \tau}{\sqrt{b^{2}-\tau^{2}}(x-\tau)} .
\end{gathered}
$$

Then we prove that, if $f(x) \in C^{1}(-b, b) ; K_{1}(x, t) \in C^{1}[(-b, b) \times(-b, b)]$, then for any $x \in(-b, b)$ the difference between solution $g(x)$ of the linear algebraic system

$$
\sum_{j=1}^{n}\left[\frac{1}{x_{i}-t_{j}}-\frac{1}{x_{i}-t_{j-1}}+h K_{*}\left(x_{i}, t_{j}\right)\right] g\left(t_{j}\right)=f^{\prime}\left(x_{i}\right), i=1, \ldots, n
$$


and the solution of eq.(4.14) tends to zero near the crack edge when $h \rightarrow 0$ (i.e. $n \rightarrow \infty)$, where the mesh nodes $x_{i}=-b+(i-1 / 2) h, i=1, \ldots, n$ and $t_{j}=-b+j h, \quad j=0,1 \ldots, n$ are taken with the constant step $h=2 b / n$.

It should be noted that this result automatically implies: if our numarically constructed solution of the system (4.16) tends to the exact one, so the constructed solution tends to zero when approaching the crack edges, as it follows from (4.14)-(4.15).

\section{Penny-Shaped Crack: Reduction to a Fred- holm Integral Equation of the Second Kind}

Here we apply a special transformation rather typical for the problems with axial symmetry.

Let us rewrite Eq.(3.9b) in the following form

$$
\int_{0}^{b} g(\rho) \rho d \rho \int_{0}^{\infty} L(s) J_{0}(r s) J_{0}(\rho s) s d s=-(1-N)^{2} \frac{\sigma_{0}}{\mu}, \quad 0<r<b,
$$

and introduce the new function $\gamma(\xi)$ as follows

$$
\int_{0}^{b} g(\rho) J_{0}(\rho s) \rho d \rho=\int_{0}^{\infty} g(\rho) J_{0}(\rho s) \rho d \rho=\int_{0}^{b} \gamma(\xi) \frac{\sin (\xi s)}{s} d \xi .
$$

The necessary and sufficient condition for Eq.(5.2) to be correct is that this must provide $g(r)=0$ for $r>b$. Let us control correctness of this statement by applying inverse Hankel transform to (5.2):

$$
\begin{gathered}
g(r)=\int_{0}^{\infty} J_{0}(r s) d s \int_{0}^{b} \gamma(\xi) \sin (\xi s) d \xi= \\
=\int_{0}^{b}\left\{\begin{array}{c}
\frac{1}{\sqrt{\xi^{2}-r^{2}}}, r<\xi \\
0, r>\xi
\end{array}\right\} d \xi=\left\{\begin{array}{cc}
\int_{r}^{b} \frac{\gamma(\xi) d \xi}{\sqrt{\xi^{2}-r^{2}}}, r<b \\
0, r>b
\end{array}\right.
\end{gathered}
$$

so (5.2) is correct. 
Now Eq.(5.1) becomes

$$
\int_{0}^{b} \gamma(\xi) \sin (\xi s) d \xi \int_{0}^{\infty} L(s) J_{0}(r s) d s=-(1-N)^{2} \frac{\sigma_{0}}{\mu}, \quad 0<r<b,
$$

and one may apply the operator

$$
A f=\int_{0}^{t} \frac{r f(r) d r}{\sqrt{t^{2}-r^{2}}}, \quad 0<t<b
$$

to the both sides of Eq.(5.4), that leads to the following integral equation with respect to the function $\gamma(\xi)$

$$
\int_{0}^{b} \gamma(\xi) d \xi \int_{0}^{\infty} \frac{L(s)}{s} \sin (\xi s) \sin (t s) d s=-(1-N)^{2} \frac{\sigma_{0} t}{\mu}, \quad 0<t<b
$$

if the applied normal load $\sigma_{0}$ is constant. When performing all above transformations, we have used the table integrals

$$
\int_{0}^{\infty} J_{0}(r s) \sin (\xi s) d s=\left\{\begin{array}{cc}
\frac{1}{\sqrt{\xi^{2}-r^{2}}}, & r<\xi \\
0 & , r>\xi
\end{array} \quad, \quad \int_{0}^{t} \frac{r J_{0}(r s) d r}{\sqrt{t^{2}-r^{2}}}=\frac{\sin (t s)}{s}\right.
$$

Let us extend the uknown function $\gamma(\xi)$ in Eq.(5.6) to a negative interval $\xi \in(-b, 0)$ as an odd function. Then the integral (5.6) can be represented as 
follows $(\delta(x)$ is the Dirac's delta)

$$
\begin{gathered}
\int_{0}^{b} \gamma(\xi) d \xi \int_{0}^{\infty} \frac{L(s)}{s} \sin (\xi s) \sin (t s) d s= \\
=\frac{1}{2} \int_{0}^{b} \gamma(\xi) d \xi \int_{0}^{\infty} L(s) \frac{\cos [(\xi-t) s]-\cos [(\xi+t) s]}{s} d s= \\
=\frac{1}{2} \int_{0}^{b} \gamma(\xi) d \xi \int_{0}^{\infty} \frac{L(s)}{s} \cos [(\xi-t) s] d s= \\
=\frac{1}{2} \int_{0}^{b} \gamma(\xi) d \xi \int_{0}^{\infty}\left[\frac{L(s)}{s}-(1-N)^{2}\left(1-c^{2}\right)\right] \cos [(\xi-t) s] d s+ \\
+\frac{\pi}{2}(1-N)^{2}\left(1-c^{2}\right) \gamma(t), \quad \text { since } \quad \int_{0}^{\infty} \cos (s \xi) d s=\pi \delta(\xi) .
\end{gathered}
$$

Therefore, in this axially symmetric problem one arrives at a very regular Fredholm integral equation of the second kind with the convolution kernel:

$$
\pi \gamma(t)+\int_{-b}^{b} \gamma(\xi) K^{*}(t-\xi) d \xi=-\frac{2 \sigma_{0} t}{\left(1-c^{2}\right) \mu}, \quad|t|<b,
$$

where

$$
\begin{gathered}
K^{*}(x)=\int_{0}^{\infty}\left[\frac{L(s)}{(1-N)^{2}\left(1-c^{2}\right) s}-1\right] \cos (x s) d s= \\
=\int_{0}^{\infty}\left[\frac{2 N c^{2} s^{2}(q-s)+(1-N)\left(1-N-c^{2}\right) q(s)}{(1-N)^{2}\left(1-c^{2}\right) q(s)}-1\right] \cos (s x) d s .
\end{gathered}
$$

Since the expression in the square brackets here is of the order $O\left(1 / s^{2}\right)$ at $s \rightarrow \infty$ (see (4.2)), it can be easily proved that the kernel $K^{*}(x)$ is regular. More precisely, it is differentiable over any finite interval, in particular $K^{*}(x) \in$ $C^{1}(-2 b, 2 b)$. 


\section{Calculation of the Stress Concentration Co- efficient}

Here we are intersted in behavior of the normal stress (which is $\sigma_{y y}$ for in-plane problem and $\sigma_{z z}$ for axially symmetric problem) near the crack edge.

Let us start from the problem with axial symmetry. We first notice that function $\gamma(\xi)$ is regular on the interval $\xi \in(-b, b)$, as a solution of the regular Fredholm equation of the second kind (5.9). Then we operate with the relation (5.4), which obviously determines the normal stress $\sigma(r, 0)$ not only for $0<$ $r<b$, but also for $r>b$. It is easily seen that with $r \rightarrow b+0$ the leading asymptotic term is given by the leading term of $L(s)$ at $s \rightarrow \infty$ :

$$
\begin{aligned}
& \frac{\sigma_{z z}(r, 0)}{\left(1-c^{2}\right) \mu} \sim \int_{0}^{b} \sin (\xi s) d \xi \int_{0}^{\infty} s J_{0}(r s) d s=-\int_{0}^{b} \gamma(\xi) \frac{d}{d \xi} \int_{0}^{\infty} \cos (\xi s) J_{0}(r s) d s= \\
=- & \int_{0}^{b} \gamma(\xi) \frac{d}{d \xi}\left\{\begin{array}{c}
\frac{1}{\sqrt{r^{2}-\xi^{2}}}, \xi<r \\
0, \xi>r
\end{array}\right\} d \xi=\int_{0}^{b} \gamma(\xi) \frac{\xi d \xi}{{\sqrt{r^{2}-\xi^{2}}}^{3}}= \\
= & \int_{0}^{b} \frac{\gamma(\xi)-\gamma(b)}{\sqrt{r^{2}-\xi^{2}}} \xi d \xi+\frac{\gamma(b)}{\sqrt{r^{2}-b^{2}}} \sim \frac{\gamma(b)}{\sqrt{r^{2}-b^{2}}} \quad \text { at } \quad r \rightarrow b+0
\end{aligned}
$$

so the stress concentration coefficient is

$$
k=\lim _{r \rightarrow b+0} \frac{\left|\sigma_{z z}(r, 0)\right|}{\mu b\left(1-c^{2}\right)} \sqrt{r^{2}-b^{2}}=\frac{|\gamma(b)|}{b} .
$$

Some examples of calculation of this dimensionless coefficient for the pennyshaped crack are shown in Figures 1 and 2.

In the plane-strain problem our approach is absolutely different, since solution of the integral equation (2.16) with the hypersingular kernel (4.9) is constructed numerically from the algebraic system (4.16), so that the applied numerical method does not operate explicitly with the root-square structure $\sqrt{b^{2}-x^{2}}$, vanishing at the edges of the crack. Therefore, we need to treat expression (2.14), which represents the normal stress $\sigma_{y y}(x, 0)$ also for $x>b$, in a direct numerical way. 
Since $K(x)=-(1-N)^{2}\left(1-c^{2}\right) /\left(\pi x^{2}\right)+O(\ln |x|), x \rightarrow 0$, it is obvious that with $x \rightarrow b+0$ the leading asymptotic term of the considered normal stress is

$$
\frac{\sigma_{y y}(x, 0)}{2 \mu\left(1-c^{2}\right)}=\frac{1}{(1-N)^{2}\left(1-c^{2}\right)} \int_{-b}^{b} g(\xi) K(x-\xi) d \xi \sim-\frac{1}{\pi} \int_{-b}^{b} \frac{g(\xi) d \xi}{(x-\xi)^{2}},
$$

so here the stress concentration factor is

$$
k=\lim _{x \rightarrow b+0} \frac{\left|\sigma_{y y}(x, 0)\right|}{\mu b\left(1-c^{2}\right)} \sqrt{x^{2}-b^{2}}=\frac{2}{\pi} \lim _{x \rightarrow b+0}\left|\int_{-b}^{b} \frac{g(\xi) d \xi}{(x-\xi)^{2}}\right| \sqrt{x^{2}-b^{2}} .
$$

Figures 3 and 4 demonstrate numerical results on computation of this dimensionless constant for some values of parameters $N, c^{2}, b=a / l_{2}$.

\section{Conclusions}

1. We have proposed a method based upon a reducing of stress concentration problem for cracks to some integral equations. In the plane-strain problem this is a hypersingular integral equation, which permits efficient direct numerical treatment. In the axially symmetric problem for penny-shaped crack, after some traditional transformations, we arrive at more regular Fredholm integral equation of the second kind. The both types of equations can generally be solved numerically.

2. In some cases the developed equations admit exact analytical solution in explicit form. The first case is for $N=0$, that is a classical linear elastic material. In this case the plane-strain problem's integral equation has only the characteristic component $-(1-N)^{2}\left(1-c^{2}\right) /\left(\pi x^{2}\right)$ and the regular part $K_{*}$ vanishes. As can be seen from Eqs.(4.15) here $N_{1} \equiv 0$, so exact solution is given by (4.14),(4.15b), that is obviously coincides with a known classical solution. For the penny-shaped crack, the kernel $K^{*}$ also vanishes, as can be directly seen from $E q .(5.9 b)$, so function $\gamma(t)$ can be explicitly extracted from Eq.(5.9a), that finally also leads to a well-known classical solution for the round plane crack.

Another simple limiting case is when parameter $b=a / l_{2}$ is small, that means the crack size to be small when compared with the physical parameter 
$l_{2}$ (the latter being of dimension of length). Indeed, in the axially symmetric problem, if $b \rightarrow 0$, then integral operator in Eq.(5.9a) vanishes. The same property takes place for the plane linear crack, since integral of the regular part of the kernel in (4.12) vanishes. Thus, in the both cases the stress-strain state near the small crack in the porous space is like in an ideally elastic classical medium, that is quite natural from the physical point of view.

3. If we investigate the influence of the porosity to the stress concentration factor shown in figures, we can discover very interesting properties. First of all, this factor in the medium with voids is always higher, under the same conditions, than in the classical elastic medium made of material of the skeleton. This can be explained with energetic arguments, since in porous media a stress, distributed near the crack edge only in the skeleton, can provide a balance of energy caused by applied normal force. So this requires more intensity of the internal stress to provide the balance.

Further, as can be seen, influence of the porosity becomes more significant for larger cracks; this is also quite natural from a physical point of view. This results is very interesting conclusion. Let us imagine a relatively small crack, which by a sufficiently high stress concentration factor extends slightly in its length. Then, as clear from the figures, the larger crack is coupled with higher stress concentration factor that causes further crack extension. This is the real physical mechanics of cracks growth in porous media. 


\section{References}

[1] M.A Biot, D.G. Willis, 'Elastic coefficients of the theory of consolidation', Journ. Applied Mech. 24 (1957) 594-601.

[2] S.C.Cowin, J.W. Nunziato, 'Linear elastic materials with voids', Journ. Elasticity 13 (1983) 125-147.

[3] M.A. Goodman, S.C. Cowin, 'A continuum theory for granular materials', Arch. Rat. Mech. Anal. 44 (1972) 249-266.

[4] M. Ciarletta, D. Iesan, Non Classical Elastic Solids, Longman, New York, 1992.

[5] A.C.Eringen, Micropolar fluids with stretch, Int.Journ. Engng.Sci. 7 (1969) 115-127.

[6] A.C.Eringen, Micropolar elastic solids with stretch, Ari Kitabevi Mathbaasi 1-18, (1971), Istanbul, Turkey (in english).

[7] I.N.Sneddon, Mixed Boundary Value Problems in Potential Theory, North-Holland, Amsterdam, 1966.

[8] A. Scalia, M.A.Sumbatyan, 'Contact problem for porous elastic halfplane', Journ. Elasticity 60 (2000) 91-102.

[9] G. Iovane, I.K. Lifanov, M.A. Sumbatyan, 'On direct numerical treatment of hypersingular integral equations arising in mechanics and acoustics', Acta Mechanica (accepted).

[10] I.S. Gradshteyn, I.M. Ryzhik, Table of Integrals, Series and Products, Academic, London, 1980. 


\section{LEGENDS TO FIGURES}

Fig 1. Relative value of the stress concentration factor $k$ with respect to

$k_{0}$ in classical elastic medium versus coupling number, penny-shaped crack: $c^{2}=0.2$.

Fig 2. Relative value of the stress concentration factor $k$ with respect to $k_{0}$ in classical elastic medium versus coupling number, penny-shaped crack: $c^{2}=0.4$.

Fig 3. Relative value of the stress concentration factor $k$ with respect to $k_{0}$ in classical elastic medium versus coupling number, plane linear crack: $c^{2}=0.2$.

Fig 4. Relative value of the stress concentration factor $k$ with respect to $k_{0}$ in classical elastic medium versus coupling number, plane linear crack: $c^{2}=0.4$. 


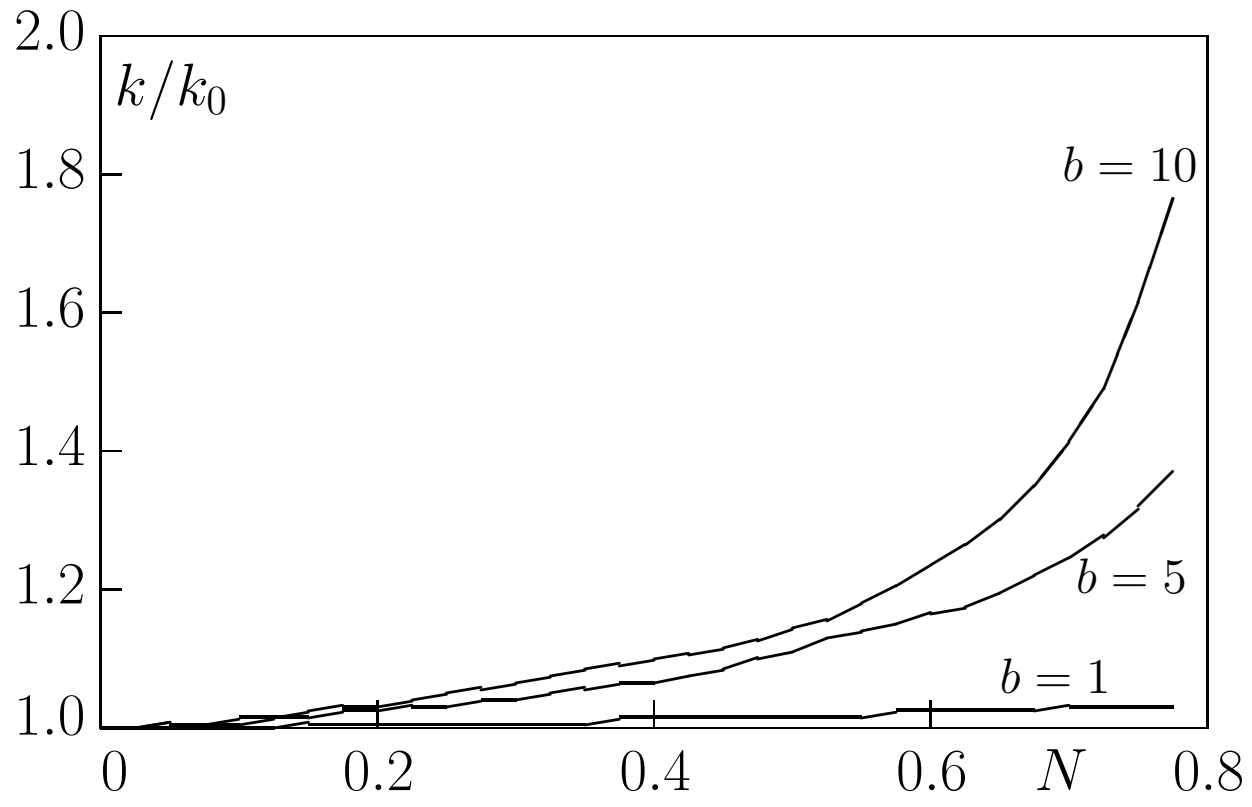

Fig.1 


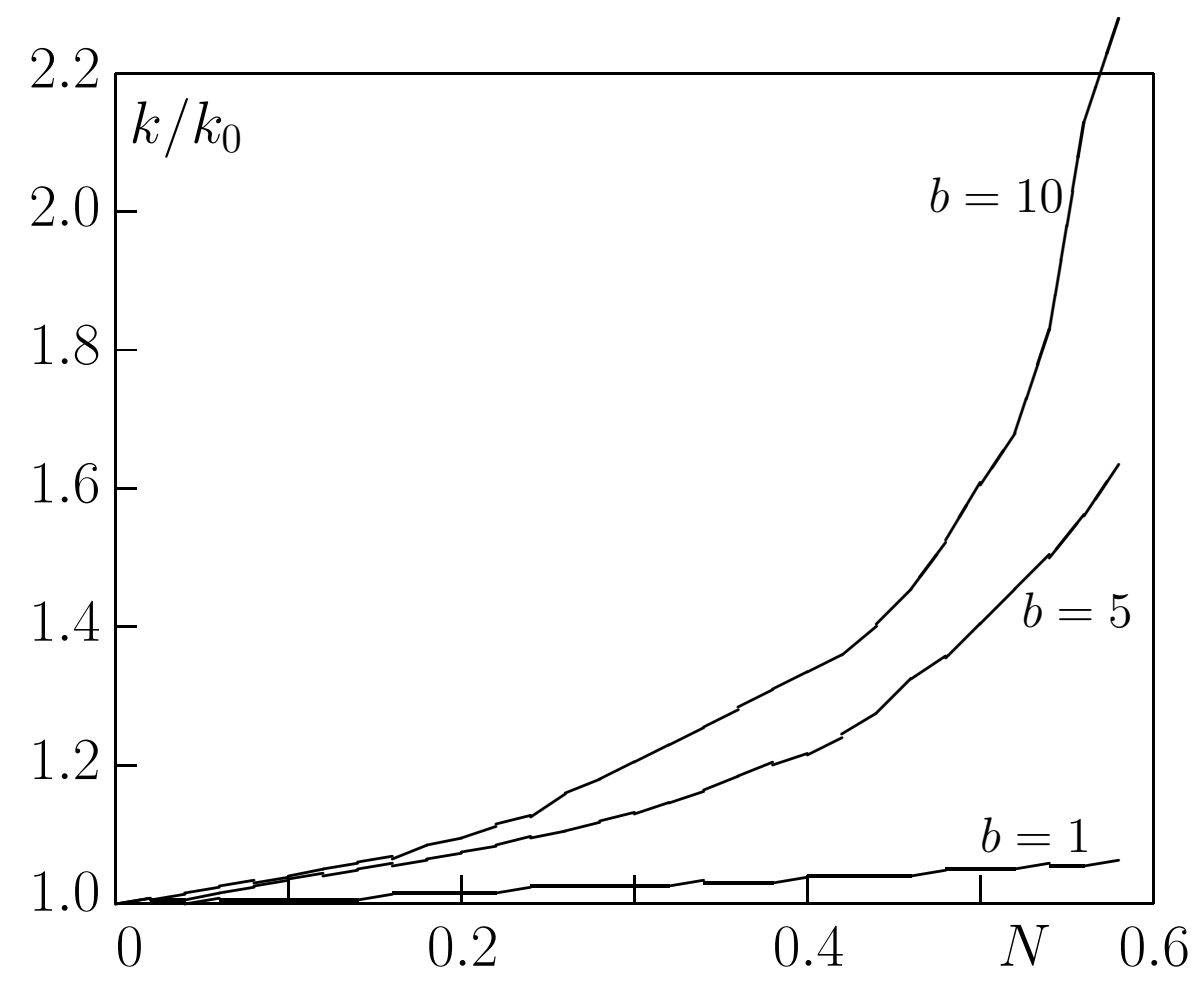

Fig.2 


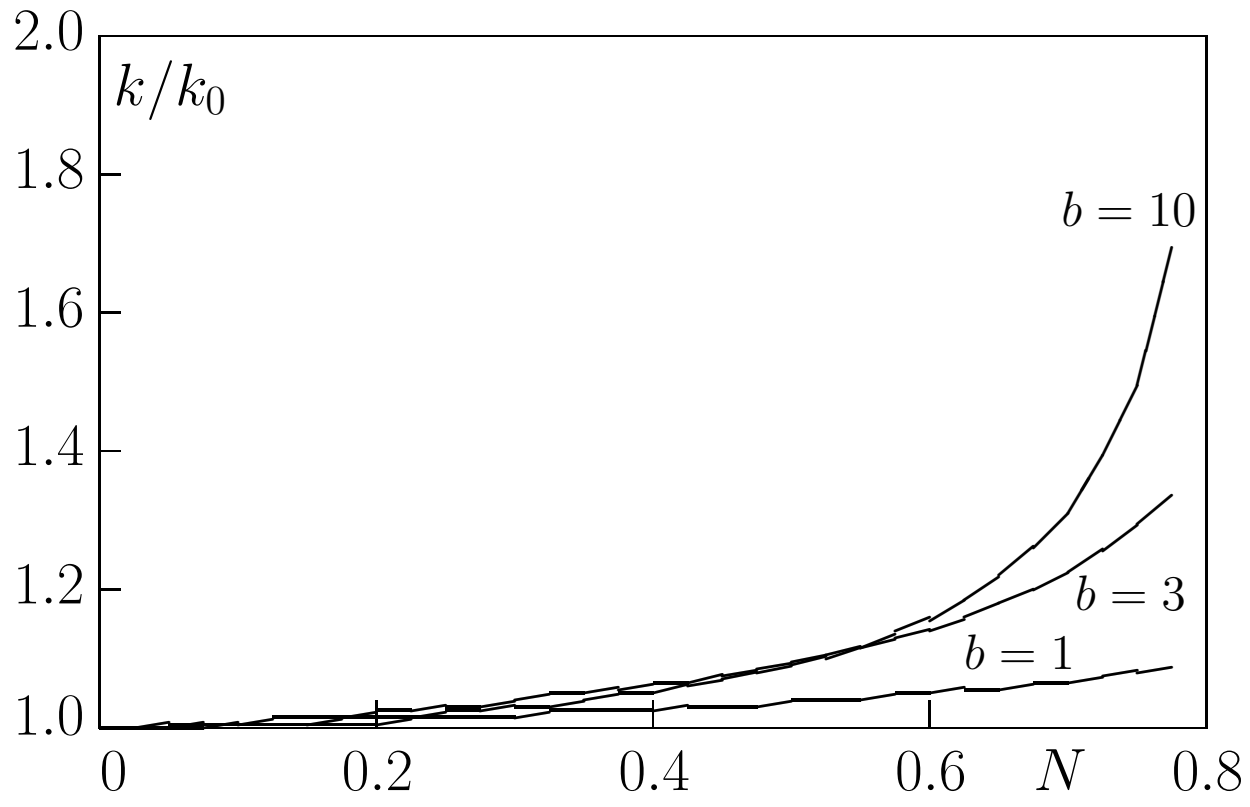

Fig.3 


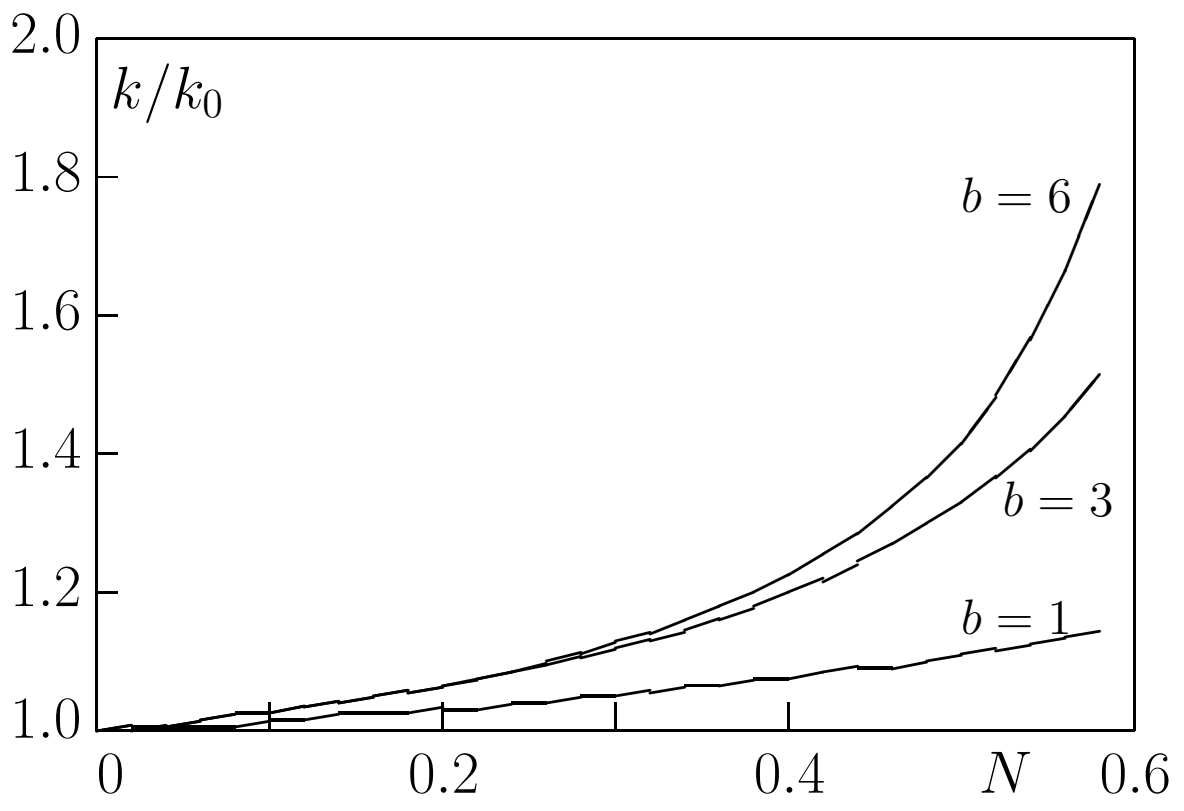

Fig.4 